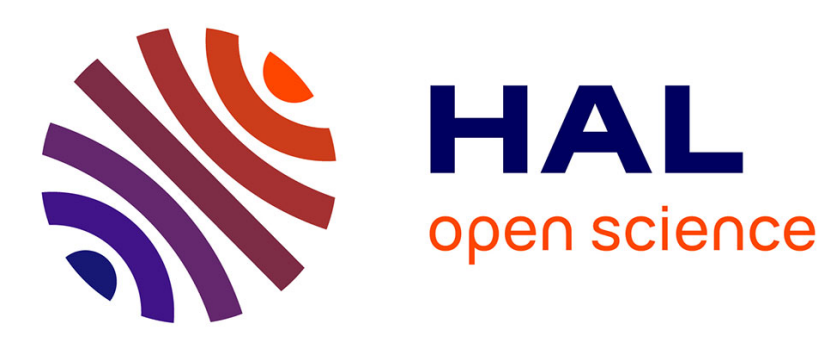

\title{
Semer des dents et planter la frontière du sauvage: le mythe thébain des Spartes
}

\author{
Karin Mackowiak
}

\section{To cite this version:}

Karin Mackowiak. Semer des dents et planter la frontière du sauvage: le mythe thébain des Spartes. Gaïa - Revue interdisciplinaire sur la Grèce archaïque, 2016, Gaïa, 19, pp.5-24. 10.3406/gaia.2016.1696 . hal-02862361

\section{HAL Id: hal-02862361 \\ https://hal.science/hal-02862361}

Submitted on 1 Dec 2021

HAL is a multi-disciplinary open access archive for the deposit and dissemination of scientific research documents, whether they are published or not. The documents may come from teaching and research institutions in France or abroad, or from public or private research centers.
L'archive ouverte pluridisciplinaire HAL, est destinée au dépôt et à la diffusion de documents scientifiques de niveau recherche, publiés ou non, émanant des établissements d'enseignement et de recherche français ou étrangers, des laboratoires publics ou privés. 


\title{
«Semer» des dents et planter la frontière du sauvage : le mythe thébain des Spartes
}

\author{
KARIN MACKOWIAK \\ Université de Franche-Comté - ISTA
}

\section{Des autochtones thébains et du sauvage*}

Comment l'autochtonie construit-elle l'idée de sauvagerie et de civilisation? Comment paramétrer la notion de «sauvage » en fonction du critère politique archaïque?

Si les aspects idéologiques et religieux de l'autochtonie ont déjà fait l'objet d'essais, essentiellement centrés sur l'Athènes classique ${ }^{1}$, le cas thébain reste, dans l'ensemble un parent pauvre de ce type d'imaginaire. Et si l'idée de «sauvage», lorsqu'on l'oppose à celle de «civilisation», a déjà été paramétrée suivant le critère de la cité, ce fut essentiellement dans les analyses concernant la littérature de la fin de l'époque archaïque, au corpus documentaire fourni ${ }^{2}$. Or, l'autochtonie thébaine, qui mérite une attention pour elle-même, présente deux spécificités : celle de constituer un discours imaginaire de la période archaïque et celle de se livrer à travers des sources fragmentaires. Son approche est donc difficile. Pourtant, il y a des pistes à explorer qui concernent les relations entre l'idée de sauvage et la cité thébaine.

La question est : comment peut-on reconstituer une vision thébaine du sauvage? Et qu'entendre par l'idée de «sauvage»?

* Ce travail a fait l'objet d'une communication orale en 2007 dans une rencontre scientifique à Besançon portant sur les frontières du sauvage.

1. Pour en rester aux ouvrages, voir Loraux (1990), Detienne (2003); Gourmelen (2004).

2. Mauduit (2006, 283-371). 
Ces interrogations nous mènent vers les origines mythiques des Thébains et les représentations qu'ils pouvaient en avoir. L'autochtonie, en Béotie, présente toutes les garanties d'un langage imaginaire comparable à celui d'Athènes. Si l'on ne dispose guère de textes «archaïques béotiens ${ }^{3}$ », les sources qui font entrevoir la vision thébaine d'un temps originel et empli de sauvage ne manquent pas : Pindare, le théâtre attique classique ou la littérature hellénistique ${ }^{4}$ ont conservé des Spartes une image haute en couleur imprégnée de culture archaïque. Reste qu'un champ lexical authentiquement thébain qui définit la «sauvagerie » ou la «civilisation» fait défaut. L'historien doit alors se tourner vers une autre méthode et raisonner en termes d'images poétiques afin de reconstituer l'idée thébaine de sauvage, ou d'une représentation équivalente. Il s'agit d'enquêter sur la façon dont un imaginaire a pu se constituer sous l'angle, notamment, d'une culture sociale et politique.

Se dévoile alors à nous une histoire : celle des Spartes, guerriers furieux issus d'un serpent non moins violent qu'eux, le fils d'Arès, qui gardait une source sur le site vierge de la future Thèbes. L'animal est tué par Cadmos qui se saisit de ses dents pour les répandre sur la terre. Des «semés», des «Spartoi» en furent engendrés qui, à peine nés, se massacrèrent aussitôt les uns les autres.

Le récit est marqué par cette naissance hors du sol originel, fulgurante et prodigieuse; une émergence effroyable aussi ${ }^{5}$ car la plupart des autochtones succombèrent aussitôt sous les coups de leurs propres frères. Cinq d'entre eux seulement survécurent. Mais ceux-là devinrent les fondateurs de Thèbes avec Cadmos, le premier roi mythique de la cité. Ces Spartes survivants épousèrent les filles de Cadmos et devinrent les ancêtres des Thébains.

3. Les sources les plus anciennes sont des fragments attribués à Stésichore d'Himère (fr 195 Page), à Hellanicos de Lesbos (FGrH IA 4 F1a-b et FGrH IA 4 F51), à Phérécyde d'Athènes (FGrH IA 3 F88 et FGrH IA 3 F22c) et quelques vers d'Eschyle (Sept contre Thèbes, 412 $414,474)$ qui ne rapportent pas tous la même version du mythe. Pour Stésichore : 'O $\mu \varepsilon \dot{v}$

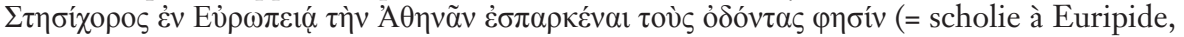

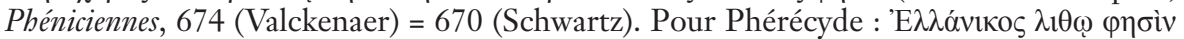

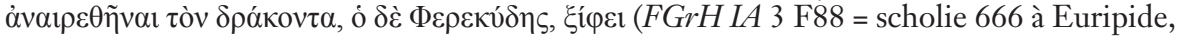

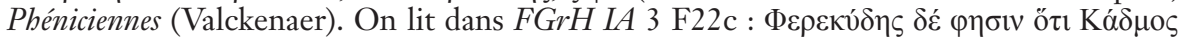

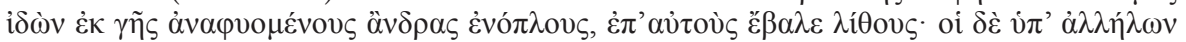

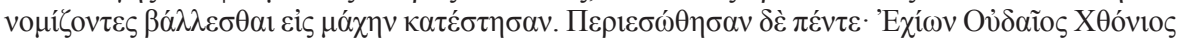

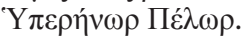

4. Il faut en effet retenir : Pindare, Hymnes, I, 1-6 (= fr. 29[5] Snell); Euripide, Phéniciennes, 638-675, 818-821 ; Apollodore, Bibliothèque, III, 4, 1; Apollonios de Rhodes, Argonautiques, III, 1179-1187. N'oublions pas certaines scholies essentielles : à Euripide, Phéniciennes, 674, 949, 666 (Valckenaer); à Lycophron, Alexandra, 1206 (Scheer); à Pindare, Isthmiques, VII, 13 (Drachmann); à Apollonios de Rhodes, III, 1179b et e (Wendel).

5. Voir scholie 675 aux Phéniciennes d'Euripide (épiphanie subite : citation infra n. 37), Apollonios de Rhodes, Argonautiques, III, 1368-1373, ou Sénèque, Edipe, 743. 
Une idée de sauvage est donc, à n'en pas douter ${ }^{6}$, présente dans ce mythe de fondation. Celle de frontières du sauvage également car un enjeu peut être ciblé dans ce récit : la naissance des Spartes se fait dans un monde sauvage ou vierge et correspond à l'émergence d'une société policée à partir d'une forme de chaos et de néant humain. Le discours mis en œuvre doit être approché sous l'angle de la valeur symbolique des images qu'il met en dynamique et qu'il rassemble avec un effet volontaire de contrastes. Or, cette approche de l'autochtonie thébaine n'est pas celle qui a prévalu dans les deux types d'approches jusqu'à présent consacrées au mythe.

Un premier point de vue a consisté à disséquer le motif des «semailles» des dents d'où les autochtones sont nés. Accompli par un héros civilisateur, le geste fut assimilé à une fécondation du sol originel et interprété comme l'introducteur de la céréaliculture ${ }^{7}$. Que le héros fondateur, Cadmos, soit responsable de l'avènement d'une ère nouvelle qui repousse les limites d'une sorte de sauvagerie primordiale ne saurait être invalidé : le mythe est celui de la fondation d'une cité; et la polis est évidemment inséparable d'un mode de vie agraire qui structure son économie et ses institutions.

Mais paramétrer le concept de sauvage sur le critère exclusif de l'alimentation appauvrit un récit dont le sens pourrait encore s'affiner par une autre mise en perspective : celle avancée par Francis Vian et Jean-Pierre Vernant ${ }^{8}$.

Ce second angle de vue s'est concentré sur l'aspect guerrier des Spartes, leur hybris et leur ressemblance avec la race de bronze hésiodique (Les Travaux et les fours, 143-155) : les autochtones thébains ont en commun avec le chalkéion génos l'arès, la folie aveugle et l'orgueil dément. Cet autre type d'approche, à la différence du premier, paramètre donc le sauvage sur le critère comportemental et l'introduit dans le registre de la moralité propre à Hésiode.

Je n'ai pas la place, ici, de critiquer plus avant l'une et l'autre démarche ${ }^{9}$ même si la dernière mérite une attention toute particulière. Au-delà des considérations relatives aux fonctions indo-européennes, les Spartes ne

6. Sur l'image du serpent et la « dévoration » sauvage incarnée par ses yeux et ses dents, voir Mauduit (2006, 146-148).

7. Se référer à Rocchi $(1989,86)$; Breglia Pulci Doria (1986, 228-230).

8. Se reporter à Vian $(1952,183)$; (1963, 158-171, 234-242) qui analysait les Spartes comme une classe militaire spécialisée de type indo-européen. Vernant (1996, 31-35 = Vernant, 1990, 28-30), qui avait comparé les Spartes à la race de bronze, excluait toute dimension agraire et prenait le contrepied de Rocchi. Pour un résumé des débats historiographiques plus anciens relatifs au caractère chthonien et guerrier des Spartes, voir Gourmelen (2004, 390-391).

9. Une étude parallèle à celle-ci, et qui propose des développements dans une perspective complémentaire (à partir de la notion de primitif) et critique (à l'égard de nos catégories de pensée) est en cours de publication dans la revue Mouseion. 
peuvent se concevoir sans un arrière-plan culturel qui guide la logique du discours et qui est surtout d'ordre politique. Cet angle de vue renouvelle l'approche des figures mythiques et nous oblige à bien réfléchir aux catégories de pensée que, par défaut, nous employons. Or, l'historien, qui se fait anthropologue, peut apporter une contribution sur ce point : les Spartes participent d'une forme ambiguë de «sauvage». Extérieurs à ce que nous appellerions «civilisation», ils semblent pourtant avoir été des paradigmes héroïques à Thèbes même où ils avaient le rôle de fondateurs.

Comment le furent-ils donc? Suivant une forme de pensée politique archaïque qui a élaboré une définition originale et complexe de ce que la pensée moderne appellerait «le sauvage». C'est ce que le présent article souhaiterait proposer : les Spartes correspondent à un discours qui dispose, en un ensemble cohérent dans une autre forme de pensée, des représentations qui rangent le «sauvage » et la monstruosité d'un côté, l'avènement de la cité et de l'humanité de l'autre... tout cela en usant d'un processus de passage. Les autochtones thébains sont donc construits suivant une vision de frontière imaginaire qui circonscrit ce qui n'est pas civilisé d'un côté, et ce qui l'est de l'autre. Reste à trouver le contenu exact de ce «sauvage» et de cette «civilisation», et cette ligne de démarcation entre deux espaces imaginaires contraires pourtant étroitement articulés entre eux. Il faut voir comment cette frontière s'exprime et sonder ses possibles origines historiques et culturelles.

\section{Sauvagerie et histoire politique : de la constitution archaïque de l'idée de frontières}

Où est donc cette frontière, comment la repérer?

Clarifions d'abord le tableau des ambiguités que nous présentent les autochtones thébains. Il y a déjà la question : comment être «sauvages», proscrits dans le monde négatif d'Arès, et fondateurs politiques tout à la fois? Et puis, les autochtones thébains ressemblent beaucoup à la race de bronze d'Hésiode et tissent donc des rapports étroits avec l'univers imaginaire de la guerre; la pousse («anaphuein $\left.{ }^{10} \gg\right)$ des Spartes n'est donc pas strictement agraire, n'appartient pas à l'univers domestiqué et paisible de Déméter. Et pourtant, l'image - marquante - d'bommes-plantes reste constamment présente à l'esprit. Un autre point de vue pourrait encore prévaloir : les héros thébains apparaissent comme les fruits d'une terre originelle qui accueille des «semailles» (avec des guillemets de nuances,

10. Phérécyde, FGrH IA 3 F22c (cité supra, n. 3) ou Euripide, Bacchantes, 540. 
dans le doute) lesquelles, sans être agraires, renvoient néanmoins à une fécondité du sol.

On ne saurait mieux définir les Spartes que comme une moisson de mort $^{11}$ : ces créatures sont issues d'un véritable espace de marge qui tronque à son paroxysme les processus courants d'émergence de la vie, qu'ils soient originels ou agraires : on ne sème pas sur de l'originel puisqu'il produit spontanément; et l'agraire n'est pas censé produire de la mort. Cette terre thébaine originelle, sauvage, est décidément étrange : vide d'humanité, elle ne s'en montre pas pour autant stérile puisqu'elle produit des hommes; féconde de monstruosité - le serpent d'Arès en est, les Spartes aussi -, elle n'en reproduit cependant pas les traits caractéristiques jusqu'au bout (puisque des hommes en émergent aussi). Ambiguités, donc.

Il faut alors se demander dans quelle mesure les Spartes ne sont pas eux-mêmes une incarnation, sorte de personnification de cette frontière entre une conception de «sauvage» et de «civilisation». Loin d'être de simples alter ego des géants ou des hommes de bronze, les Spartes dessinent une image vraiment très nuancée dans cette mare de sang qui macule une terre à la fois sauvage et matrice de la civilisation. Les autochtones thébains semblent appartenir à un monde de l'entre-deux, situés à la fois dans les marges et au centre de la cité. Mais de quelle manière? C'est ce qu'il faut commencer par voir.

Les autochtones allient à un comportement d'bybris, monstrueux, une dimension humaine puisqu'il s'agit d'hommes ancestraux. Or, c'est la principale manifestation d'une situation intermédiaire entre un espace de marge et un autre de civilisation dont il faut observer de près la manière dont ils s'emboîtent l'un dans l'autre. Les analyses qui se sont intéressées aux Spartes ne les ont approchés que sur un plan globalisant : elles n'ont vu que leur promotion à la civilisation quand ce n'est pas, à l'inverse, leur arès qui a été relevé à l'exclusion du reste. Les recherches présentent donc le défaut d'avoir voulu homogénéiser les autochtones thébains ${ }^{12}$. Mais une lecture plus attentive des sources laisse entrevoir des différences à l'intérieur de ce groupe d'hommes-plantes : d'un côté se trouvent les victimes du massacre fratricide, les Spartes qui, à l'instar de la race de bronze, ne survivent pas; de l'autre côté se trouvent les survivants. Les textes en font régulièrement mention dans la mesure où ces derniers étaient pourvus de noms : il y avait,

11. Tel que cela ressort du sacrifice de Ménécée, autre descendant mythique des Spartes qui doit, à l'instar de ses ancêtres, rendre le sang à la terre selon Euripide, Phéniciennes,

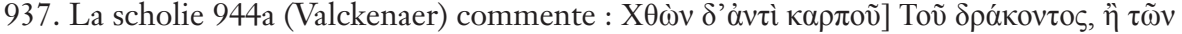

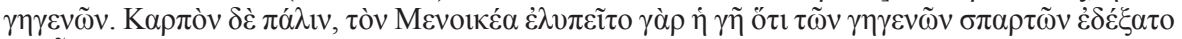

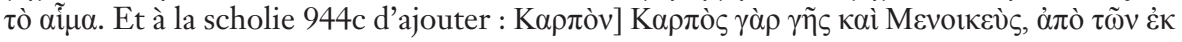

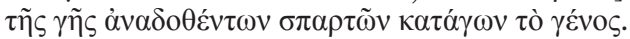

12. Sur ce type d'approche, voir Gourmelen (2004, 391-393) ou Detienne (2003, infra n. 25). 
par exemple, Échion (littéralement «le serpent») ou Hyperénor. Ceux-là sont devenus les gendres de Cadmos et ont eu des descendants ${ }^{13}$. À la différence de leurs frères morts, emportés par leur propre bybris, ce groupe de Spartes est fondamentalement associé à l'ancestralité et à la société politique. Issus de dents d'un monstre, ces survivants ont finalement pu accéder à l'humanité, là où précisément l'autre partie des autochtones a échoué, race avortée, cantonnée dans une monstruosité sans retour.

Cette multitude anonyme de Spartes morts, éphémères, est donc la seule à présenter des analogies strictes avec la race de bronze hésiodique. Elle se bat : c'est tout ce qu'elle sait faire et ce pourquoi elle est née. Elle sombre aussitôt dans la mort et l'oubli ${ }^{14}$. Les Spartes trépassés correspondent alors à une monstruosité totale, paramétrée sur une pratique effrénée de la guerre, vision rédhibitoire et finalement synonyme de chaos : ces créatures chthoniennes font en effet se confondre dans un même mouvement la naissance et la mort ${ }^{15}$.

L'objectif du discours et sa complexité, alors, se clarifient : à l'intérieur des Spartes se trouve bien une frontière imaginaire entre l'avènement de la civilisation, rangée du côté de l'humanité et de la cité, et le chaos rejeté de l'autre côté, avec la monstruosité et la guerre sans limites - en un mot une forme de sauvagerie. Le récit insiste sur le fait que les survivants ont été rares; il faut donc comprendre que ceux-là ont été extirpés de cette sauvagerie. Il y a dans ce détail la marque d'une fonction attribuée aux fondateurs autochtones : les survivants plantent sur la terre thébaine les frontières d'une catégorie du sauvage paramétrée sur les pratiques de la guerre. Les Spartes fondateurs passent de l'intérieur à l'extérieur d'une sauvagerie laquelle se construit autour d'une réflexion sur l'intégration et l'exclusion de la société politique. Ce sont des préoccupations typiques des communautés de l'époque archaïque - ce que les historiens appellent les «cités»-qui ont, à travers cet imaginaire, fait œuvre d'une forme de pensée politique. Certes, les Spartes, en tant que «jumeaux» de la race de bronze hésiodique, s'envisagent aussi dans un univers poétique plus large. Mais, en tant que fondateurs de la cité, ils appartiennent en propre à la culture thébaine. Ce contexte a sans doute contribué à élaborer l'idée selon laquelle la frontière du sauvage, à Thèbes, est construite sur des critères sociaux et comportementaux.

13. Penthée est fils d'Échion d'après Euripide, Bacchantes, 264-265, 541-542, 995-996, 1018-1019, 1030, repris par Pausanias, Périégèse, IX, 2, 4. Créon est fils d'Hyperénor d'après la scholie 674 aux Phéniciennes d'Euripide (Valckenaer).

14. À comparer avec Les Travaux et les fours, 152-155.

15. À propos des guerriers colques nés des dents du serpent gardien de la toison d'or, Apollonios de Rhodes, Argonautiques, 1396-1398, précise comment les autochtones meurent sans avoir totalement pu émerger du sol, expressions du chaos d'un mouvement à la fois ascendant et descendant. 
En face de cet imaginaire archaïque, l'historien doit alors poser une autre question : la manière dont cette frontière du sauvage a pu se constituer avec le temps.

Malgré leurs spécificités, les Spartes appartiennent à un patrimoine mythologique de Béotie centrale et évoluent à la fois dans le giron de l'épopée archaïque et dans l'histoire sociale et religieuse de la région.

Qu'une idée de sauvage et de ses frontières se soit constituée à partir d'une réflexion archaïque sur la société, la ressemblance des Spartes avec d'authentiques guerriers homériques le confirme. Les héros survivants sont imprégnés de valeurs aristocratiques et, contrairement à la race de bronze qui meurt dans l'anonymat le plus total, les cinq cofondateurs de Thèbes sont spécifiquement pourvus de noms. Le détail est fondamental car il dissocie les autochtones survivants de la tradition hésiodique pour l'agréger à une autre, épique et thébaine. Les noms les plus intéressants demeurent «Pélôros», qui renvoie à une taille physique exceptionnelle, qualité essentielle du guerrier homérique, et «Hyperènôr », littéralement celui qui dépasse les autres, le plus fort par l'épée ${ }^{16}$.

Ces figures imaginaires semblent exprimer l'idéal de vie des basileis glorifiés par l'Iliade. Mais elles ne sont pas seulement les héritières d'une culture de «classe » qu'on a tendance à appeler «aristocratique ${ }^{17} »$. Les Spartes semblent aussi pourvus des caractères communautaires propres aux poleis archaïques. En effet, les sources insistent sur les armes dont ils étaient revêtus dès leur naissance : le casque et le bouclier reviennent le plus souvent. Est-ce un renvoi aux armes défensives typiques du soldatcitoyen ${ }^{18}$ ? Il est difficile de l'affirmer. Les guerriers mythiques sont certes décrits en groupe, mais ils s'inscrivent aussi, et de manière essentielle, dans le prolongement de l'univers héroïque. Les autochtones thébains ont un caractère bigarré, partagé entre un cadre imaginaire épique régional et un autre de type civique qu'on aurait tort d'opposer l'un à l'autre ${ }^{19}$.

16. Voir par exemple : Apollodore, Bibliothèque, III, 4, 1-2 ; Hellanicos, FGrH IA 4 F1a-b; Pausanias, Périégèse, IX, 5, 2-6; Phérécyde, FGrH IA 3 F22c, sans oublier deux fragments attribués à Eschyle, 731a et b (Mette) ou les scholies à Apollonios de Rhodes, Argonautiques, III, 1179b et e (Wendel), à Pindare, Isthmiques, I, 41 (Drachmann) et à Euripide, Phéniciennes, 674 et 949 (Valckenaer).

17. Sur l'usage de ces notions, voir Duplouy (2006, 37-43, 75-77).

18. Sur la $\pi \alpha v 0 \pi \lambda i ́ a$ des Spartes, voir par exemple la scholie 676 aux Phéniciennes d'Euripide (Valckenaer). Leurs armes sont encore mentionnées dans Phérécyde, FGrH IA 3 F22c, dans les scholies aux Phéniciennes d'Euripide 675 (Valckenaer), aux Sept contre Thèbes d'Eschyle 412a (Langwitz Smith) ou aux Argonautiques d'Apollonios de Rhodes III, 1179b (Wendel). Les vers 796 et 939 des Phéniciennes, commentés par les scholies 802 et 946 (Valckenaer) insistent respectivement sur leurs boucliers et casques. Vian (1963, 227-228 passim) fait également valoir leurs lances.

19. On rappellera le point de vue de Morris $(2000,176-177)$ : la technique de la phalange hoplitique n'est pas synonyme du déclin des valeurs héroïques. Sur la cité dans Homère, voir Haubold (2005, 25-48). 
À une époque où les cités sont plus que jamais «aristocratiques», ces traits distinctifs poussent à voir en ces personnages une élite sociale imaginaire : les Spartes apparaissent comme une synthèse d'héritages poétiques du haut-archaïsme mêlés à des formes d'évolutions sociale et communautaire.

En ce qui concerne l'histoire sociale et politique de la Béotie, l'historien note alors trois types de données. (i) Certaines thèses plaident en faveur d'une constitution précoce des poleis en Grèce centrale ce qui pourrait rattacher les autochtones au début de l'époque archaïque, au guerrier homérique qui, fût-il individuel, est en même temps décrit comme le protecteur d'une forme de cité, ou de communauté humaine archaïque déjà élaborée : Hector en est le meilleur exemple. Ces paramètres pourraient nous mener au $\mathrm{IX}^{\mathrm{e}}$ siècle ou, au plus tard, au VIII ${ }^{\mathrm{e}}$ siècle av. J.-C. ${ }^{20}$. (ii) Les données de l'archéologie funéraire issues de la même région permettent peut-être d'affiner le tableau : le mobilier de la tombe $\mathrm{n}^{\circ} 6$ du prince d'Érétrie traduit l'héroïsation traditionnelle d'un chef de guerre récupéré par la cité vers la fin du VIII siècle av. J.-C. Les Spartes fondateurs pourraient s'être forgés dans ce contexte de resserrement varié entre l'élite sociale et les communautés, autour du cœur de l'âge archaïque ${ }^{21}$. (iii) Enfin, lorsqu'on songe à la forte critique par Hésiode des guerriers pleins d'arès incarnés par la race de bronze, on ne peut s'empêcher d'attribuer à ces figures de sauvagerie guerrière, pour une période peut-être située au début du VIIe siècle av. J.-C., une grande maturité, celle-là même qui fait polémique dans les Travaux et les Fours. Entre les Spartes fondateurs et le chalkéion génos se noue donc certainement une problématisation sociale dont l'enjeu, exprimé par le langage poétique, tourne autour de l'autorité morale et politique des paradigmes épiques.

Les Spartes fondateurs sont les faux-jumeaux des hommes de bronze : à Thèbes les guerriers autochtones sont positivés. On n'y partageait pas le même point de vue qu'à Ascra où le même profil mythologique de guerriers était jugé excessif, rejeté par Hésiode ${ }^{22}$. Reste qu'à Thèbes se lisent peut-être aussi des nuances par rapport au modèle du guerrier excessif qui n'est pas vraiment pris comme modèle en tant que tel. Les Spartes, finalement, établissent une frontière qui circonscrit le sauvage à l'intérieur

20. Voir Edwards (2004, 168-169) dans la suite de Buck (1979, 87-88) ou de Morris $(1998,12)$. 21. Si l'on suit les analyses de Polignac (1996, 31-40) et de Morris (2000, 273, 287-289, 306), les rites et offrandes funéraires des élites évoluent, ont tendance à s'homogénéiser ce qui pourrait correspondre à un resserrement entre les catégories sociales élevées et les communautés situées en arrière-plan, entre le $\mathrm{VIII}{ }^{\mathrm{e}}$ et le $\mathrm{VII}{ }^{\mathrm{e}}$ siècle. L'héroïsation du prince de la tombe ${ }^{\circ} 6$ d'Érétrie ou celle des défunts des tombes 1, 2 et 79 de Salamine de Chypre vient confirmer cette évolution : se référer à Morris (1999, 63-70) ou à Duplouy, Mariaud \& De Polignac (2010, 280-290). Sur les fouilles à Érétrie, consulter Bérard (1970, 31-32, 68-71) et Blandin (2007, 111-114).

22. Travaux personnels en cours sur une comparaison approfondie entre mythes thébain et hésiodique, une question qui rejoint celle de l'écart entre Homère et Hésiode. 
d'une pratique guerrière rédhibitoire (incarnée par les autochtones qui meurent de leurs propres excès) tandis que, en dehors de cette sauvagerie, émerge l'humanité et une autre pratique de la guerre, intégrée dans la cité : de l'autre côté de cette ligne de démarcation se trouvent les cinq fondateurs. En même temps, ces derniers séparent désormais l'aristocrate de ce qu'il n'est plus : un professionnel de guerre isolé dans ses intérêts du reste de la communauté. Il demeure donc probable que les autochtones thébains soient situés à mi-parcours chronologique entre le début de la période archaïque et l'époque d'Hésiode : c'est là, selon toute vraisemblance, le contexte historique de leur fonction paradigmatique pour Thèbes.

C'est ainsi que le mythe en vient à construire, édifier une nouvelle frontière du sauvage au cœur de l'époque archaïque. Les Spartes en sont une véritable personnification dont les enjeux et le sens ${ }^{23}$ ne semblent pas avoir été totalement mis en valeur par leurs commentateurs. Le rôle premier des fils du serpent d'Arès est bien d'établir ce qui apparaît, au fond, comme une ligne de rupture entre ordre et désordre social, en organisant ce discours autour du comportement guerrier paradigmatique, intégrable à la cité, ou, au contraire, sauvage, c'est-à-dire à proscrire hors de la cité. La fonction de fondateur des Spartes et leurs ambiguités prennent finalement sens dans cette autre forme de pensée, par le biais d'une confrontation entre un groupe humanisé et un autre monstrueux. Il faut repérer ces deux ensembles différents dans la mesure où ils construisent une alternative qui peut être moralisée, voire même une sorte d'antithèse susceptible d'opposer, in fine, deux modèles comportementaux.

Les Spartes héroïques, ni exclusivement positivés comme cela pourrait ressortir des vers d'Eschyle, ni exclusivement péjoratifs comme cela ressort des vers d'Euripide ${ }^{24}$, semblent en fait avoir été modelés pour incarner une frontière. Dans sa dimension sociale, cette frontière se lit comme un pivot ou un point de rupture à partir duquel l'homme guerrier bascule dans les formes condamnables d'une violence sauvage, contraire à l'ordre sociopolitique. Loin d'être exclusivement des créatures de sang et de meurtre comme l'affirmait Marcel Detienne ${ }^{25}$, ces figures que l'on pourrait - à tort - globaliser comme des monstres humains ou hommes monstrueux, contrairement aux apparences, ne sont en rien interchangeables entre elles : des deux groupes de Spartes surgit une véritable mise en regard qui traduit une forme de

23. Sur l'importance du regard du destinataire dont dépend la création (ou recréation) des personnifications littéraires ou iconographiques, voir Brog (2002, 49-80).

24. Dans Eschyle, les descendants des Spartes, Mélanippe et Mégareus sont positivés (Sept contre Thèbes, 412-141, 473-474). Dans Euripide, les Spartes apparaissent sous leur plus mauvais jour : voir Suppliantes, 578 ou Phéniciennes, 129, 240, 820-822 ou 1008.

25. Detienne (2003, 80-81, 97, 118-119) met en valeur les Spartes du point de vue d'une souillure ineffaçable pour Thèbes, une position trop unilatérale qui plaque le point de vue athénien sur les autochtones thébains. 
pensée politique, un choix de comportement social que l'élite du temps, prise comme modèle des projections imaginaires, semblait incarner.

L'horizon imaginaire et poétique édifie donc une frontière d'une idée du sauvage qui fait correspondre à une ligne de rupture entre humanité et monstruosité une autre qui sépare ordre et désordre social. Sans doute peut-on encore approfondir l'enquête en nous tournant vers d'autres éléments culturels de la région. Car l'horizon rituel peut à son tour affiner le propos : il nous permet de mieux saisir le terreau culturel qui a vu naître ces créatures et qui a produit sa propre définition du sauvage.

\section{Sauvagerie et stabilité sociale : plaidoyer pour une frontière-passage}

Il reste à évoquer d'autres figures avec lesquelles les Spartes partagent des points communs : les Courètes. Eux aussi portent l'équipement militaire, notamment le casque et le bouclier ${ }^{26}$. Ils appartiennent à l'univers des mystères de Cybèle et sont, comme les Spartes, des guerriers imaginaires. De même, ces figures étaient populaires en Béotie : les Courètes sont par exemple représentés sur un beau relief de Livadia du III ${ }^{\mathrm{e}}$ siècle av. J.-C. ${ }^{27}$.

Cette sculpture est, certes, tardive : sans prétendre identifier les Spartes d'époque archaïque aux Courètes hellénistiques, le rapprochement des autochtones thébains avec les serviteurs de Cybèle peut être signifiant du point de vue de la logique suivant laquelle les Spartes furent compris par les Béotiens.

L'image d'hommes-plantes interpelle : elle évoque un processus qui fait évoluer ces créatures depuis le monde souterrain jusqu'à la lumière, le même qui, d'après notre analyse, fait passer les Spartes de la sauvagerie et la monstruosité à la civilisation et à l'humanité. Les autochtones pourraient, de même, renvoyer à des croyances du type de celles attestées pour des cultes à mystères, notamment la nécessité d'effectuer un passage. Or, l'idée selon laquelle il faut contrôler le sauvage, le circonscrire au moyen d'une domestication des pratiques de la guerre, ne rejoint-elle pas ce genre de représentation? Ensuite, l'idée de frontière est consubstantielle à celle de passage : la question se pose bien de savoir dans quelle mesure les Spartes n'en furent pas une métaphore poétique.

Or, le sanctuaire dionysiaque du Cabirion, situé sur le territoire de Thèbes, apporte quelques pistes de réflexion.

26. Des analogies déjà retenues par Usener (1894, 461-471), reprises par Bianchi (1976, 2). D'autres positions sont plus prudentes : voir Borgeaud (1996, 60, 96), Jeanmaire (1939, 593-608) ou Voutiras (1996, 243-256).

27. Musée national d'Athènes (inv. 3942) : voir la description de Daumas (1998, 76-77, pl. X, 1). 
Une série de vases à figures rouges des $\mathrm{V}^{\mathrm{e}}$ et $\mathrm{IV}^{\mathrm{e}}$ siècles av. J.-C. fut découverte sur les lieux. Une peinture (fig. 1) parmi bien d'autres montre un jeune homme en train d'accomplir des exercices guerriers sous l'égide du dieu Cabire, représenté assis ou comme Dionysos banquetant (fig. 2).

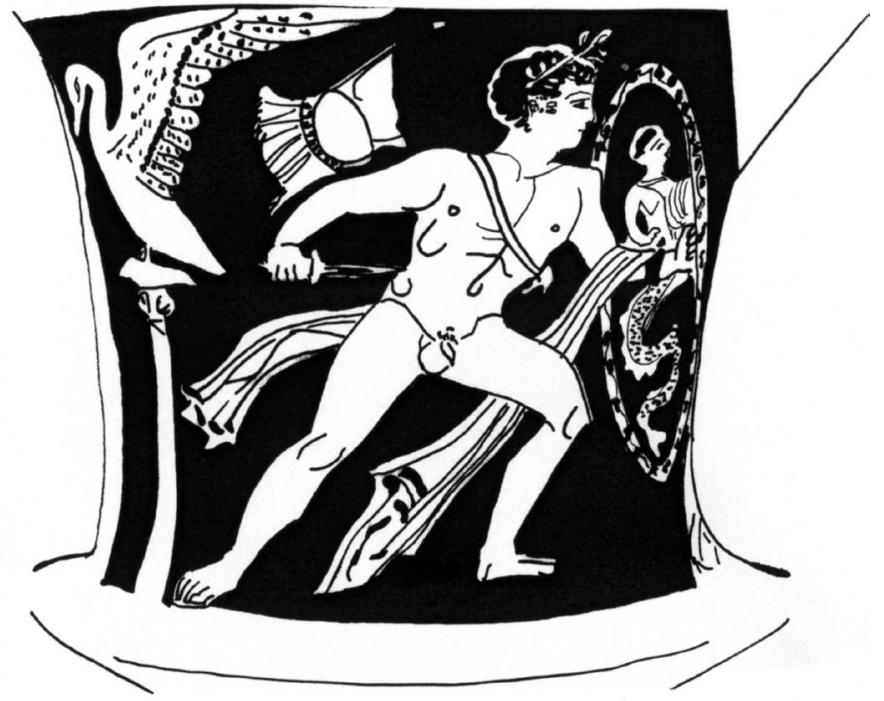

Fig. 1 - Cantare du Musée national d'Athènes, Inv. 12486. Époque classique. Dessin de K. Mackowiak d'après Daumas (1998, pl. V, 2).

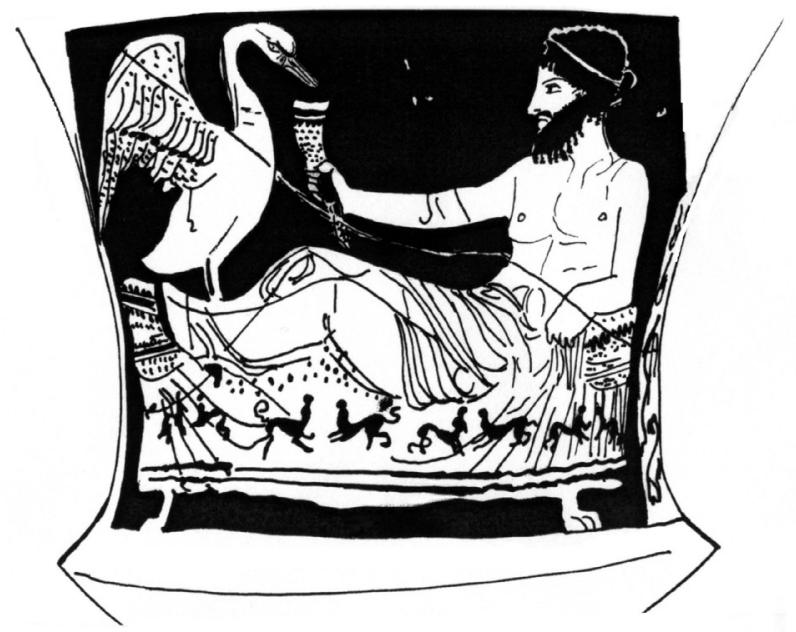

Fig. 2 - Cantare du Musée national d'Athènes, Inv. 12487. Époque classique. Dessin de K. Mackowiak d'après Daumas (1998, pl. VII, 2). 
Sur d'autres documents, les jeunes gens, hoplites, cavaliers aux vêtements ouvragés ou auriges, s'élancent toujours de la même manière ou procèdent à des manipulations d'armes. On ne peut confirmer s'il s'agit là de rites ou de joutes en armes comme celles qui accompagnent l'initiation des jeunes gens aux mystères cabiriques de Pergame. Le matériel archéologique découvert permet tout au plus d'affirmer que le Cabirion thébain accueillait favorablement les jeunes hommes en âge de porter les armes ${ }^{28}$.

Par ailleurs, les sources disponibles tendraient à confirmer que l'initiation au sanctuaire était, à l'époque classique, compartimentée suivant le critère sexuel ${ }^{29}$ et sans doute celui sociologique. La taille exceptionnelle ainsi que la qualité artistique des vases montrent que les offrandes au dieu n'étaient peut-être pas exclusivement - mais sans doute prioritairement le fait de personnages riches, semblables à ceux qui apparaissent sur les fameuses stèles funéraires en pierre noire (fig. 3) : ces guerriers intrépides sont toujours représentés richement vêtus et, de surcroît, dans une posture analogue à celle des jeunes hommes sur les vases ${ }^{30}$.

L'état des sources ne permet pas de dire si ces manifestations rituelles et croyances existaient déjà sous cette forme à l'époque archaïque quand s'est formé le mythe des Spartes. Mais, compte tenu de l'antique rapport de l'élite sociale avec les armes et plus encore de l'évolution historique de l'obtention des droits politiques à Thèbes, fortement inégalitaires encore au moment de la guerre du Péloponnèse ${ }^{31}$, la formation militaire des jeunes gens a pu constituer, au moins jusqu'à l'âge classique, une tradition sociologiquement marquée et favoriser un élitisme d'obédience archaïque, celui que l'on retrouve précisément dans le mythe ${ }^{32}$.

L'histoire des fondateurs autochtones participe pleinement de la vivacité, à Thèbes, d'une arétè guerrière dont les contours archaïques se sont comme figés dans les monuments culturels ainsi que dans les pratiques concrètes : que l'on songe, par exemple, à l'usage du char dans les corps d'élite thébains du $\mathrm{V}^{\mathrm{e}}$ siècle av. J.-C. ${ }^{33}$, ou au fameux bataillon sacré constitué de trois cents hommes cantonnés sur l'acropole et contemporain d'Épaminondas. Euripide, dans les Suppliantes, 702, parle de ce régiment comme celui des «hommes des Dents-du-Dragon» où il est aisé de voir l'empreinte des

28. Pour des informations sur Pergame, voir Jones (1974, 199). Pour les vases thébains mentionnés, voir Daumas (1998, 96-104 et pl. V, VI, VII).

29. Se référer à Daumas $(1998,83)$.

30. Sur ces stèles, voir Daumas (1998, 195-200 ainsi que fig. 43-44) ou Daumas (1995, 285-309). Sur l'initiation d'aristocrates grecs et romains aux mystères de Samothrace, voir Daumas (1998, 224-225, 250) et Collini (1990, 261).

31. Sur l'importance de la fortune pour l'obtention des droits politiques à Thèbes et l'évolution de la société thébaine depuis l'époque archaïque, voir Salmon (1978, 49-59).

32. À propos des Spartes ancêtres des rois et des princes thébains, voir supra n. 11 et 13 .

33. Pour l'épisode de Délion, se référer à Diodore de Sicile, Bibliothèque historique, XII, 70, 1. 


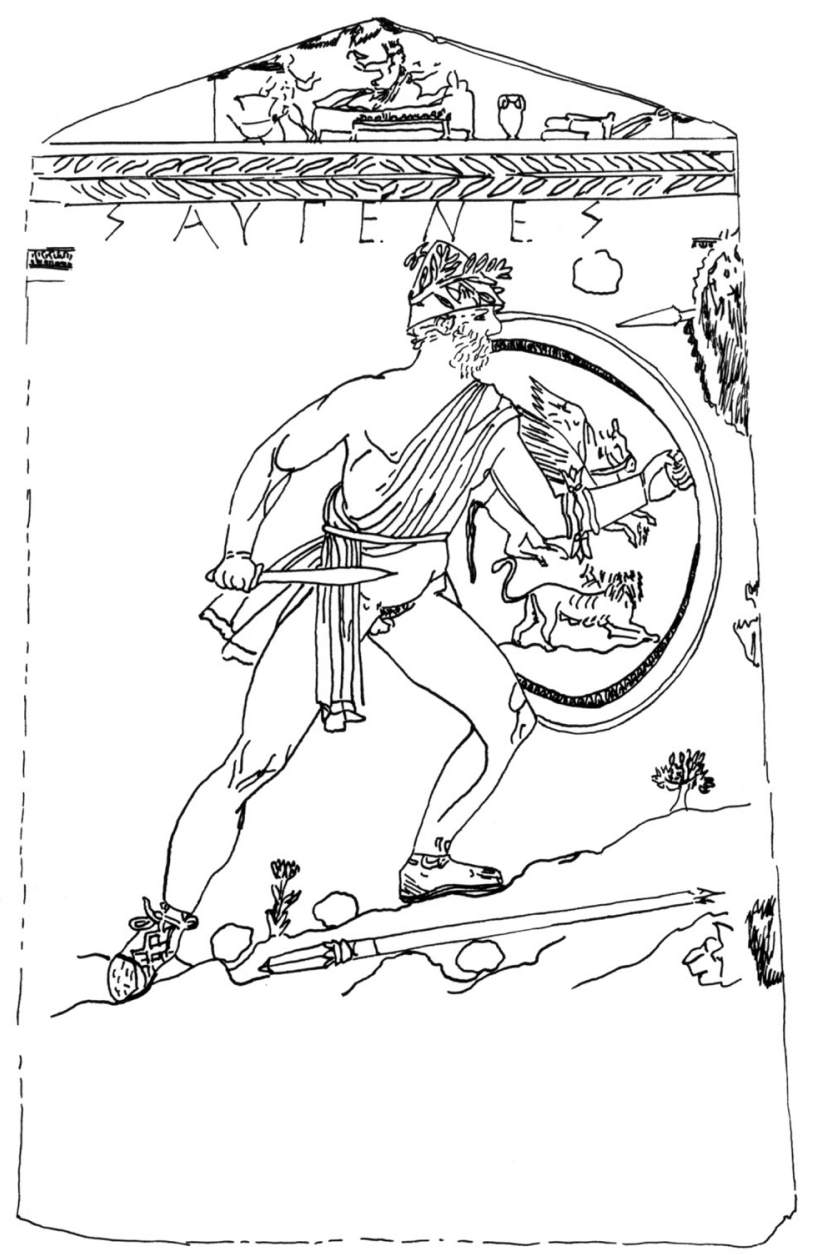

Fig. 3 - Stèle en pierre noire du Musée de thèbes, Inv. 56. Début du IV siècle av. J.-C. Dessin de K. Mackowiak d'après Daumas (1998, fig. 44, 216).

Spartes. Si les textes à notre disposition sont, là encore, tardifs ${ }^{34}$, la raison d'être de ce corps d'élite pourrait bien renvoyer à l'existence d'un groupe de guerriers ouvert à quelques individus peut-être initiés, répondant au modèle des frairies guerrières typiques des sociétés traditionnelles ${ }^{35}$.

34. Voir Plutarque, Pélopidas, 18, 1. Le bataillon sacré n'a peut-être pas existé à l'époque archaïque sous la forme évoquée par le moraliste mais l'existence antérieure de corps d'élite ne fait pas de doute.

35. Sur les confréries initiatiques prenant pour modèle des serviteurs mythiques comme les Courètes, voir Dacosta (1991, 31-35). Sur le rapprochement des Spartes aux géants 
Rien ne permet de dire que telle initiation, si elle a existé, ait eu lieu au Cabirion mais les références culturelles communes au mythe des Spartes et au sanctuaire, qu'il s'agisse de ce maintien d'un élitisme d'ancien style ou de certaines représentations de passage, ne laissent pas de réfléchir.

Aussi les curieuses «semailles» de dents par Cadmos, écartées de la connotation agraire attendue qui ne sont pas vraiment la leur, ne pourraient-elles pas être situées à la confluence de représentations guerrières et dionysiaques? Ce geste qualifie d'abord des semailles sauvages, un oxymore qui se résorbe dans l'idée d'un jaillissement surnaturel d'une végétation dans un espace de marge ${ }^{36}$. Cet angle de vue revient à supposer que le mythe des Spartes pourrait avoir été constitué à partir du même type de représentations imaginaires que celles déployées à l'époque postérieure, sur les vases classiques où Dionysos, confondu au dieu Cabire, se trouve mêlé à l'univers d'Arès.

Ces questions en amènent d'autres, notamment celles du sens profond de ces hommes-plantes qui poussent frénétiquement dans un espace sauvage : ne paraissent-ils pas intégrables dans des représentations de frontièrespassage qui concernent un certain nombre de jeunes gens prêts à intégrer le monde d'Arès? Si la bipartition des Spartes en un groupe humain et un autre monstrueux paraît orientée vers la nécessité de définir une pratique socialisée (et humanisée) de la guerre contre une autre sauvage, leur épiphanie de la terre ${ }^{37}$, si semblable à une anodos, ne pourrait-elle pas renvoyer au passage d'un statut à un autre?

Les représentations d'émergences verticales depuis le sol peuvent se comprendre, au moins dans notre cas, comme une métamorphose des formes ou de la nature. Les Spartes nés de dents de dragon répandues sur la terre ne sont, à la base, rien d'autre qu'une nouvelle manifestation morphologique de ce même serpent dont ils partagent le caractère chthonien et l'agressivité, une sauvagerie animale qui ressort du qualificatif thèrios

et sur leur caractère tribal ou de confrérie guerrière, voir Vian (1952, 186-187) et Vian (1963, 169-171).

36. Le geste de l'éparpillement des dents d'une créature d'Arès sur le sol rappelle le lancer rituel de membres d'animaux dans les croyances de fécondité dionysiaques : voir HalmTisserand (2004, 126-131). Sur la proximité de Dionysos et Déméter à Thèbes, se reporter à Moreux $(1970,1-14)$.

37. À propos des Phéniciennes d'Euripide décrivant «une vision d'hommes en armes à la

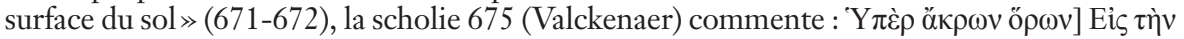

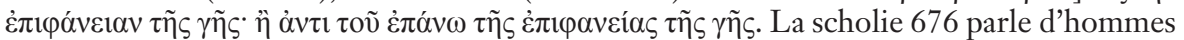
produits à la lumière hors de la terre, oi ỏv $\alpha \varphi \alpha v \varepsilon ́ v \tau \varepsilon \varsigma \grave{\varepsilon} \kappa \gamma \tilde{\eta} \varsigma$ ou d'une vision d'armures

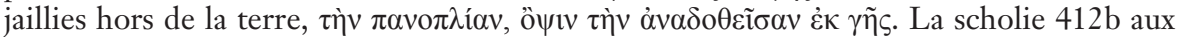

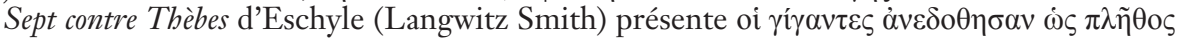

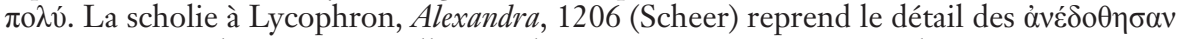

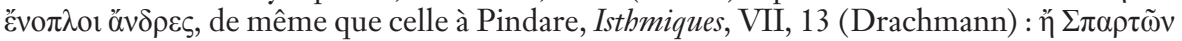

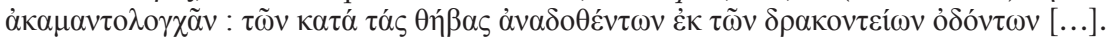


employé à leur égard ${ }^{38}$. On peut alors se rappeler le sens du nom d'Échion, l'un des cinq survivants ${ }^{39}$. Du point de vue d'une manifestation à peine différenciée d'une créature d'arès, les Spartes qui sont en train de jaillir de la terre présentent donc, à ce stade de leur naissance, une anthropomorphie toute illusoire dans le miroir de leur comportement monstrueux. Le sauvage épouse de façon paroxystique la forme de ces créatures où la monstruosité l'emporte dans un premier temps sur l'humanité avant que ce rapport ne s'inverse. Ce peut être là le résultat discursif d'une représentation de passage et d'une certaine conception de l'héroïsme qui forge sa logique propre, s'immisce dans un univers chthonien et guerrier pour en modifier les traits traditionnels : les Spartes les plus forts, les survivants, ne sont plus monstrueux : ayant réussi une épreuve et un passage, ils deviennent les plus braves, les plus accomplis au terme d'une pratique de la guerre qui fait d'eux des fondateurs, des êtres intégrés dans la polis.

Les Spartes semblent donc représenter une forme de réflexion sur l'être guerrier : le processus d'émergence chthonien fait d'eux des créatures-frontières, ni serpent ni homme (dans un premier temps), et me paraît pouvoir être mis en relation avec les chemins de l'accomplissement de l'individu guerrier, celui de son aboutissement graduel compris en termes d'idéal et d'éducation militaires. Là où le sauvage se définit comme opposition à une pratique socialisée de la guerre, il peut également se définir par opposition à une initiation ou une éducation. D'une frontière-pivot/rupture qui établit une ligne de partage entre guerre sauvage et guerre socialisée, à la frontièrepassage qui établit un seuil d'intégration du guerrier à la communauté, il n'y a qu'un pas : l'une est complémentaire de l'autre ${ }^{40}$.

Il n'y aurait rien d'étonnant à trouver dans la Thèbes archaïque ce genre de représentations culturelles et imaginaires à une époque où l'individu formé était autant initié qu'il était éduqué ${ }^{41}$. L'image des «semailles» ellemême me paraît centrale dans cette forme de logique qui construit des frontières autant à l'échelle des individus qu'à celle du monde. Sauvages, ces étranges «semailles» sur un espace de marge bientôt appelé à devenir un centre de civilisation, sont en même temps héroïques; de celles qui paraissent indiquer, à partir des types de fécondation symbolique du sol,

38. La scholie 827 aux Phéniciennes (Valckenaer) explique cette hérédité en termes savants :

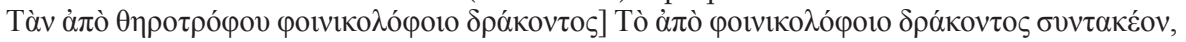

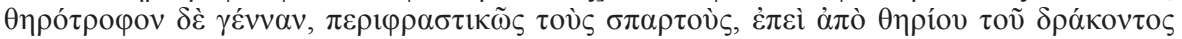
$\dot{\varepsilon} \gamma \varepsilon v v \eta ́ \theta \eta \sigma \alpha v[\ldots]$. La férocité animale entendue par «thèrios» revient dans la scholie 668

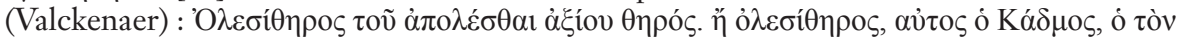
$\theta \tilde{\eta} \rho \alpha$ ò $\lambda \dot{\varepsilon} \sigma \alpha \varsigma$ [...]. Sur la métamorphose comme apanage du serpent, parce qu'il change de peau, voir Goyens-Slezakowa (1992, 135-152).

39. Supra, p. 10.

40. Voir les remarques fondatrices de Van Gennep (1991, 109, 115, 121).

41. Sur ces deux notions, voir Redfield (2003, 255-259). 
une idée de renaissance de l'être. Sur le plan de l'ordre social fondant la cité, il n'est pas interdit d'approcher telle renaissance à partir d'une nécessité : celle de l'intégration du monde d'Arès, sauvage et furieux, dans la cité. Cette résorption d'un haut degré de violence dans la polis ne se conçoit pas sans le passage nécessaire du guerrier de son état initialement sauvage à celui éduqué, ce qui lui donne accès à l'héroïsme.

\section{Quelques déductions sur la conception thébaine du sauvage}

Sans procéder obligatoirement du rite ni sans accoucher en sens inverse de ce dernier ${ }^{42}$, il me paraît plus sûr d'affirmer que le mythe a contribué à fixer, voire à figer, des frontières sociocomportementales. Qu'entendre par là ?

Faire des Spartes les personnifications d'une frontière de la sauvagerie aux enjeux sociaux (séparation ordre-désordre) et quasi taxinomiques (séparation humanité-monstruosité), pose les bases d'une forme de pensée différente de la nôtre. L'écart se lit tout particulièrement de la notion de sauvage qui ne se réduit pas à nos conceptions d'évolution ou de la civilisation. Pour revenir au premier type d'approche historiographique dont le mythe d'autochtonie avait fait l'objet, celui de l'avènement de la céréaliculture, ce genre de raisonnement plaque implicitement sur le récit une vision des origines contemporaines de notre mode de pensée : le passage en termes temporels, d'un mode de vie sauvage (sous-entendu ignorant l'agriculture) vers un autre censé être plus évolué. Le scénario qui se structure sur le critère alimentaire s'appuie alors en même temps sur un autre, technique, synonyme de progrès matériel, et sur une conception du temps qui mériterait de plus amples développements ${ }^{43}$.

Il est peu probable que l'idée thébaine du sauvage ait fonctionné de la sorte. Il convient d'insister sur la capacité du mythe à restructurer des images topiques (comme celle des géants) pour définir une idée de sauvage dans le cadre d'un jeu syntaxique au caractère fallacieux : en lieu et place de l'opposition graduée entre humanité sauvage et humanité civilisée agit bien un autre écart : celui qui peut séparer les individus d'une même société. La représentation des frontières qu'imposent les Spartes se comprend dans cette logique de pensée où la graduation se place à l'échelle de l'individu lui-même et où la conscience historique de l'évolution d'une société et de ses conceptions des limites est substituée par des seuils successifs de rupture qui jalonnent le parcours éducatif (ou initiatique?) du guerrier. On retiendra ici que les Spartes, qu'ils soient susceptibles de renvoyer à une initiation ou à une éducation, correspondent dans l'un et l'autre cas à la

42. Pour une synthèse sur la question du mythe et du rite, voir par exemple Segal (1998) ou Versnel (1993, chap. 1, 88).

43. Voir supra, n. 9. 
volonté d'une société de canaliser le sauvage identifié, in fine, à une forme condamnable de la violence.

S'agissant de conscience historique, l'autre intérêt du mythe d'autochtonie réside dans la fluctuation historique des frontières du sauvage. Il y a dans le cycle fondateur de Thèbes une autre curiosité : l'existence de deux récits des origines. Aux Spartes s'ajoute en effet l'histoire des Antiopides, Amphion et Zéthos, mentionnés dès l'Odyssée, XI, 260-265 comme les constructeurs des murailles de Thèbes. Or, une forme de continuité s'établit entre les deux cycles : construire des frontières et de maîtriser la violence, contenir la sauvagerie occupent en effet les deux mythes. La réponse apportée par l'une et l'autre histoires est tout simplement différente.

Là où les jumeaux établissent une frontière physique entre le sauvage et la cité en édifiant des remparts - ils constituent un discours et des représentations imaginaires différentes, peut-être plus anciennes que le mythe d'autochtonie ${ }^{44}$-, les Spartes établissent une frontière autre que monumentale à l'intérieur même de la cité, une frontière qui paraît être circonscrite à l'intérieur de chaque individu constitutif de la cité aristocratique. Le fameux bataillon sacré laisse pensif à cet égard : il a pu entretenir la popularité du mythe des Antiopides, cantonné comme il l'était sur l'acropole de la Cadmée. Mais surtout il a pu reproduire dans la réalité ce principe de frontière contre le sauvage déjà incarné par les Spartes : les trois cents guerriers d'exception incarnaient le principe même de murailles inébranlables qui, toujours, protègeraient la cité : à l'époque classique, ils ont pu en constituer une forme métaphorique. Constitué d'érastes et d'éromènes, on connaît la solidarité indéfectible de ces soldats dont la rupture des rangs était réputée exceptionnelle ${ }^{45}$. C'est en perpétrant l'image de la frontière contre le sauvage qu'Épaminondas et Caphisodoros trouvèrent la mort ensemble ${ }^{46}$. De ce raid chaotique, il est permis de penser que les Thébains l'aient perçu comme la ligne de front d'une forme de sauvagerie menaçant la cité et devant laquelle les soldats thébains en général - et le bataillon sacré en particulier - se sont dressés comme un rempart imparable.

Les Spartes, Amphion et Zéthos montrent que les anciens Thébains avaient conceptualisé une idée du sauvage et de sa frontière par récits successifs, ou différents. C'est la preuve d'une véritable préoccupation sociale et politique qui a modelé l'imaginaire à Thèbes. De l'époque archaïque jusqu'au désastre

44. Amphion le musicien et Zéthos le fort ont fait l'objet d'une approche que j'ai présentée dans une autre contribution : «Les mythes fondateurs de Thèbes et l'histoire : les mises en formes du passé d'une cité et leurs enjeux» (Guelfucci, 2011).

45. Se reporter à la digression de Plutarque, Pélopidas, 18, 2-7.

46. Sur cet épisode symptomatique des liens indéfectibles entre certains guerriers thébains, voir Plutarque, Dialogues sur l'amour, 761c. Sur l'appartenance d'Épaminondas à «la race des Spartes», consulter Pausanias, Périégèse, VIII, 11, 8. 
de l'invasion macédonienne, les fils d'Arès semblent bien avoir continué à bâtir autour de la société humaine les frontières qui la protégeaient de la sauvagerie non sans intégrer pour autant la violence dans la cité, l'une de ses formes du moins. Ce problème n'a pas dû être évident à résoudre compte tenu de la nécessité des cités naissantes de se prémunir contre la violence qui les menaçait mais, en même temps, d'en user pour survivre.

De ce point de vue, les Spartes disent finalement un déplacement historique décisif à Thèbes de la frontière du sauvage, voire une transcendance de celle-ci. Intégrant le sauvage dans la cité, ce dernier devait s'en trouver nécessairement transformé, mis sous le contrôle de la société, résolvant peut-être ainsi le problème homérique de la violence sociale tel qu'il apparaît dans l'Iliade et encore dans l'Odyssée.

Quelle place donner à la violence guerrière? Comment la réguler et en faire un facteur de stabilité sociale? Les Spartes paraissent pleinement répondre à une question sinon philosophique du moins essentielle, de l'essence des choses et des êtres qui fait de la frontière du sauvage, à Thèbes, un monument méconnu de la pensée politique et une muraille à l'abri du temps qui passe, fichée dans la terre comme devait l'être dans les esprits l'éternel sens du monde.

\section{BIBLIOGRAPHIE}

BÉRARD Claude, L'hérôon à la porte de l'ouest. Eretria, 3, Berne, A. Francke, 1970.

BIANCHI Ugo, The Greek Mysteries, Leyde, Brill, 1976.

BLANDIN Béatrice, Pratiques funéraires d'époque géométrique à Érétrie. Espace des vivants, demeure des morts. Eretria, 17, Lausanne, École suisse d'archéologie en Grèce, 2007.

BORGEAUD Philippe, La mère des dieux. De Cybèle à la vierge Marie, Paris, Seuil, 1996.

BREGLIA PULCI DORIA Luisa, «Miti di Demetra e storia beotica», Dialogues d'bistoire ancienne, 12, 1986, p. 217-240.

BROG Barbara, Der Logos des Mythos. Allegorien und Personnifikationen in der frühen griechischen Kunst, Munich, Fink, 2002.

BUCK Robert J., A History of Boiotia, Edmonton Alberta, The University of Alberta Press, 1979.

COLLINI Paolo, «Gli Dèi Cabiri di Samotracia: origine indigena o semitica?», Studi Classici e Orientali, 40, 1990, p. 237-287.

DACOSTA Yves, Initiations et sociétés secrètes dans l'Antiquité gréco-romaine, Paris, Berg International, 1991.

DAUMAS Michèle, «Des stèles de Thèbes au Gortyte de Vergina : images

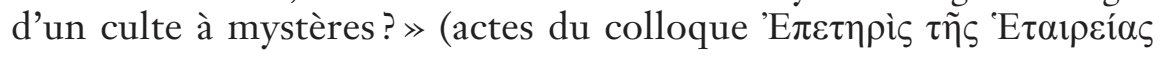




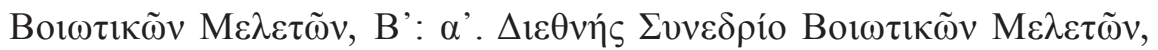

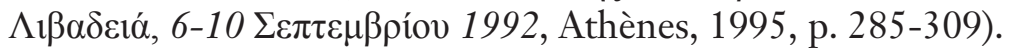

—, Cabiriaca. Recherches sur l'iconographie du culte des Cabires, Paris, De Boccard, 1998.

DETIENNE Marcel, Comment être autochtone. Du pur Athénien au Français raciné, Paris, Seuil, 2003.

DUPLOUY Alain, Le prestige des élites. Recherches sur les modes de reconnaissance sociale en Grèce entre les $X^{e}$ et $V^{e}$ s. av. F.-C., Paris, Les Belles Lettres, 2006.

—, MARIAUD Olivier \& POLIGNAC François de, «Les sociétés grecques», dans R. Étienne (éd.), La Méditerranée au VII ${ }^{e}$ s. av. F.-C. (essais d'analyses archéologiques), Paris, De Boccard, 2010, p. 275-309.

EDWARDS Anthony T., Hesiod's Ascra, Berkeley - Los Angeles - Londres, University of California Press, 2004.

GOURMELEN Laurent, Kékrops, le roi-serpent. Imaginaire athénien, représentations de l'humain et de l'animalité en Grèce ancienne, Paris, Les Belles Lettres, 2004.

GOYENS-SLEZAKOWA Christine, «L'initiation dans le Philoctète de Sophocle», dans L'initiation (actes du colloque international de Montpellier, 11-14 avril 1991), Montpellier, université Paul Valéry, 1992, 2, p. 135-152.

HALM-TISSERAND Monique, «Le sparagmos, un rite de magie fécondante», Kernos, 17, 2004, p. 119-142.

HAUBOLD Johannes, «The Homeric Polis», dans M. Herman Hansen (éd.), The Imaginary Polis. Symposium, Fanuary 7-10, 2004, Copenhague, Copenhaguen Polis Centre, 2005, p. 25-48.

JEANMAIRE Henri, Couroi et Courètes. Essai sur l'éducation spartiate et sur les rites d'adolescence dans l'Antiquité bellénique, Lille, Bibliothèque universitaire de Lille, 1939.

JONES Christopher P., «Diodoros Pasparos and the Nikephoria of Pergamon», Chiron, 4, 1974, p. 183-205.

LESKY Albin, «Herakles und das Ketos», Anzeiger der Österreichischen Akademie der Wissenschaften. Philosophisch-Historische Klasse, 104, 1967, p. 1-6.

LORAUX Nicole, Les enfants d'Athéna : idées athéniennes sur la citoyenneté et la division des sexes, Paris, Maspero, 1990.

MACKOWIAK Karin \& GUELFUCCI Marie-Rose, «Les mythes fondateurs de Thèbes et l'histoire : les mises en formes du passé d'une cité et leurs enjeux», dans M.-R. Guelfucci (dir.), Feux et enjeux de la mise en forme. Aux marges de l'bistoire, Besançon, 29/11-02/12/2006, Besançon, 2011 (DHA, suppl. 4.2), p. 563-589.

MAUDUIT Christine, La sauvagerie dans la poésie grecque d'Homère à Eschyle, Paris, Les Belles Lettres, 2006. 
MOREUX Bernard, «Déméter et Dionysos dans la septième Isthmique de Pindare $\gg, R E G, 83,1970$, p. 1-14.

MORRIS Ian, «Archaeology and Archaic Greek History», dans N. Fisher \& H. Van Wees (éd.), Archaic Greece: New Approaches and New Evidence, Londres, Duckworth, 1998, p. 1-91.

—, «Iron Age Greece and the Meanings of 'Princely Tombs'», dans Les princes de la protobistoire et l'émergence de l'État (actes de la table ronde internationale organisée par le Centre Jean Bérard et l'École française de Rome, Naples, 27-29 octobre 1994), Naples-Rome, Centre Jean Bérard, 1999, p. 63-70.

—, Archaeology as Cultural History. Words and Things in Iron Age Greece, Oxford, Blackwell, 2000.

POLIGNAC François de, «Entre les dieux et les morts. Statut individuel et rites collectifs dans la cité archaïque», dans The Role of Religion in the Early Greek Polis (proceedings of the Third International Seminar on Ancient Greek Cult, organized by the Swedish Institute at Athens, 16-18 October 1992), Stockholm, P. Aström, 1996, p. 31-40.

REDFIELD James, «Initiations and Initiatory Experience», dans D. B. Dodds \& C. A. Faraone (éd.), Initiations in Ancient Greek Rituals and Narratives: New critical Perspectives, Londres -New York, Routledge, 2003, p. 255-259.

ROCCHI Maria, Kadmos e Harmonia. Un matrimonio problematico, Rome, L'Erma di Bretschneider, 1989.

SALMON Pierre, Étude sur la confédération béotienne (447/6-386). Son organisation et son administration, Bruxelles, Palais des Académies, 1978.

SEGAL Robert A., The Myth and Ritual Theory. An Anthology, Oxford, Blackwel, 1998.

USENER Hermann, «Pasparios», Rheinisches Museum, 49, 1894, p. 461-471.

VAN GENNEP Arnold, Les rites de passage [1909], Paris, Picard, 19913.

VERMEULE Emily, Aspects of Death in Early Greek Art and Poetry [1979], Berkeley - Los Angeles - Londres, University of California Press, 1984³.

VERNANT Jean-Pierre, Mythe et pensée chez les Grecs. Études de psychologie bistorique, $I$ [1965] Paris, La Découverte, 1996 .

— \& VIDAL-NAQUET Pierre, La Grèce ancienne 1. Du mythe à la raison, Paris, Seuil, 1990.

VERSNEL Henk S., Transition and Reversal in Myth and Ritual, Leyde, Brill, 1993.

VIAN Francis, La guerre des géants. Le mythe avant l'époque bellénistique, Paris, Klincksieck, 1952.

—, Les origines de Thèbes. Cadmos et les Spartes, Paris, Klincksieck, 1963.

VOUTIRAS Emmanuel, «Un culte domestique des Corybantes», Kernos, 9, 1996, p. 243-256. 